\title{
A Note on Generalized Weighted Arithmetic Mean Summability Factors via Quasi-B-Power Increasing Sequence
}

\author{
Ripendra Kumar \& Aditya Kumar Raghuvanshi \\ Department of Mathematics IFTM University.Moradabad, U.P.,India, 244001
}

Abstract: In this paper we have established a theorem on generalized summability factors via quasi- $\beta$-power increasing sequence, which gives some new results and generalizes some previous known results.

Keywords: Weighted arithmetic mean summability, summability, summability factors and quasi- $\beta$-power increasing sequence.

Mathematical classification : 40D15, 40F05, 40G99.

\section{Introduction:}

Let $\Sigma a_{n}$ be a given infinite series with partial sums $\left\{s_{n}\right\}$. We denote by $u_{n}^{\alpha}$ and $t_{n}^{\alpha}$ the $n$-th Cesaro means of order $\alpha$, with $\alpha>-1$, of the sequence $\left(s_{n}\right)$ and $\left(n a_{n}\right)$ respectively such that

$$
\begin{aligned}
& u_{n}^{\alpha}=\frac{1}{A_{n}^{\alpha}} \sum_{v=0}^{n} A_{n-v}^{\alpha-1} s_{v} \\
& t_{n}^{\alpha}=\frac{1}{A_{n}^{\alpha}} \sum_{v=0}^{n} A_{n-v}^{\alpha-1} v a_{v}
\end{aligned}
$$

where $A_{n}^{\alpha}=O\left(n^{\alpha}\right), \alpha>-1, A_{0}^{\alpha}=1$ and $A_{-n}^{\alpha}=0$ for $n>0$.

A series $\Sigma a_{n}$ is said to be summable $|C, \alpha|_{k}, k \geq 1$. If (FLETT [7]).

$$
\sum_{n=1}^{\infty} n^{k-1}\left|u_{n}^{\alpha}-u_{n-1}^{\alpha}\right|^{k}=\sum_{n=1}^{\infty} \frac{\left|t_{n}^{\alpha}\right|^{k}}{n}<\infty
$$

and $\Sigma a_{n}$ is said to be summable $|C, \alpha, \delta|_{k}, k \geq 1$ and $\delta \geq 0$ if (FLETT [7]).

$$
\sum_{n=1}^{\infty} n^{\delta k-1}\left|\mathrm{t}_{n}^{\alpha}\right|^{k}<\infty
$$

Let $\left(p_{n}\right)$ be a sequence of positive numbers such that

$$
P_{n}=\sum_{v=0}^{n} p_{v} \rightarrow \infty \text { as } n \rightarrow \infty\left(P_{-i}=p_{-i}=0, i \geq 1\right)
$$

The sequence to sequence transformation

$$
\sigma_{n}=\frac{1}{P_{n}} \Sigma p_{v} s_{v}
$$

defines the sequence $\left(\sigma_{n}\right)$ of the Weighted arithmetic mean or simply $\left(\bar{N}, p_{n}\right)$ mean of the sequence $\left(s_{n}\right)$ generated by the sequence of coefficients $\left(p_{n}\right)$ (HARDY [8]). The series $\Sigma a_{n}$ is said to be summable $\left|\bar{N}, p_{n}\right|_{k}, k \geq 1$ if (BOR [7])

$$
\sum_{n=1}^{\infty}\left(\frac{P_{n}}{p_{n}}\right)^{k-1}\left|\Delta \sigma_{n-1}\right|^{k}<\infty
$$

and it is said to be summable $\left|\bar{N}, p_{n}, \delta\right|_{k} ; k \geq 1$ and $\delta \geq 0$ if (BOR [3]) 


$$
\sum_{n=1}^{\infty}\left(\frac{P_{n}}{p_{n}}\right)^{\delta k+k-1}\left|\Delta \sigma_{n-1}\right|^{k}<\infty
$$

where $\Delta \sigma_{n-1}=\sigma_{n}-\sigma_{n-1}=\frac{p_{n}}{P_{n} P_{n-1}} \sum_{v=1}^{n} p_{v-1} a_{v} ; n \geq 1$.

In the special case $P_{n}=1, k=1$ and $\delta=0$ for all values of $n,\left|\bar{N}, p_{n}, \delta\right|_{k}$ summability is reduces to $\left|\bar{N}, p_{n}\right|$-summability.

A positive sequence $\left(b_{n}\right)$ is said to be almost increasing if there exist a positive increasing sequence $\left(c_{n}\right)$ and two positive constants $A$ and $B$ such that -

$$
A c_{n} \leq b_{n} \leq \beta c_{n}
$$

A positive sequence $\left(\gamma_{n}\right)$ is said to be quasi- $\beta$-power increasing sequence if there exist a constant $k=k(\beta, \gamma) \leq 1$ such that-

$$
k n^{\beta} \gamma_{n}=m^{\beta} \gamma_{m}
$$

Hold for all $n \geq m+1$. It should be noted the every almost increasing sequence is a quasi- $\beta$-power increasing sequence for any non-negative $\beta$ and converse is not true.

\section{Known Results:}

BOR [2] has proved the following theorem for $\left|\bar{N}, p_{n}\right|_{k}$-summability factors.

Theorem 2.1: Let $\left(X_{n}\right)$ be a positive non-decreasing sequence and let there be sequences $\left(\beta_{n}\right)$ and $\left(\lambda_{n}\right)$ such that

$$
\begin{gathered}
\left|\Delta \lambda_{n}\right| \leq \beta_{n} \\
\beta_{n} \rightarrow 0 \text { as } n \rightarrow \infty \\
\sum_{n=1}^{\infty} n\left|\Delta \beta_{n}\right| X_{n}<\infty \\
\left|\lambda_{n}\right| X_{n}=\mathrm{O}(1)
\end{gathered}
$$

If

$$
\sum_{v=1}^{n} \frac{\left|t_{v}\right|^{k}}{v}=\mathrm{O}\left(X_{n}\right) \text { as } n \rightarrow \infty
$$

where $\left(t_{n}\right)$ is the $n$-th $(C, 1)$ mean of the sequence $\left(n a_{n}\right)$ and $\left(p_{n}\right)$ is the sequence such that

$$
\begin{aligned}
P_{n} & =\mathrm{O}\left(n p_{n}\right) \\
P_{n} \Delta p_{n} & =\mathrm{O}\left(p_{n} p_{n+1}\right)
\end{aligned}
$$

then the series $\sum_{n=1}^{\infty} \frac{a_{n} P_{n} \lambda_{n}}{n p_{n}}$ is summable $\left|\bar{N}, p_{n}\right|_{k}, k \geq 1$.

Later BOR [4] has generalized Theorem 2.1 for the $\left|\bar{N}, p_{n}, \delta\right|_{k}$ summability factors.

Theorem 2.2: Let $\left(X_{n}\right)$ be a positive non-decreasing sequence and the sequence $\left(\beta_{n}\right)$ and $\left(\lambda_{n}\right)$ are such that the condition (2.1) - (2.7) of Theorem (2.1) are satisfied with condition (2.5) replaced by

$$
\sum_{v=1}^{n}\left(\frac{P_{v}}{p_{v}}\right)^{\delta k} \frac{\left|t_{v}\right|^{k}}{v}=\mathrm{O}\left(X_{n}\right) \text { as } n \rightarrow \infty \text {. }
$$

If 


$$
\sum_{n=v+1}^{m+1}\left(\frac{P_{n}}{p_{n}}\right)^{\delta k-1} \frac{1}{P_{n-1}}=\mathrm{O}\left(\left(\frac{P_{n}}{p_{n}}\right)^{\delta k} \frac{1}{P_{v}}\right) \text { as } m \rightarrow \infty .
$$

Then the series $\sum_{n=1}^{\infty} \frac{a_{n} P_{n} \lambda_{n}}{n p_{n}}$ is summable $\left|\bar{N}, p_{n}, \delta\right|_{k} k \geq 1$ and $0 \leq \delta \leq \frac{1}{k}$.

Recently BOR [5] has proved the following theorem.

Theorem 2.3: Let $\left(X_{n}\right)$ be an almost increasing sequence. If the condition (2.1)-(2.4) and (2.6)-(2.9) are satisfied then the series $\sum_{n=1}^{\infty} a_{n} \frac{P_{n} \lambda_{n}}{n p_{n}}$ is summable $\left|\bar{N}, p_{n}, \delta\right|_{k}, k \geq 1$ and $0 \leq \delta<\frac{1}{k}$.

\section{Main Results:}

The aim of this paper is to prove the Theorem 2.3 under more weaker conditions for this we use the concepts of quasi- $\beta$-power increasing sequence. Now we shall prove the following theorem.

Theorem 3.1: Let $\left(X_{n}\right)$ be quasi- $\beta$-power increasing sequence if the condition (2.1)-(2.4) and (2.6)-(2.9) are satisfied, then the series $\sum_{n=1}^{\infty} a_{n} \frac{P_{n} A_{n}}{n p_{n}}$ is summable $\left|\bar{N}, p_{n}, \delta\right|_{k}, k \geq 1$ and $0 \leq \delta<\frac{1}{k}$.

\section{Lemma:}

We need the following lemma for the proof of Theorem 3.1.

Lemma 4.1: (LEINDER [9]) Under the condition on $\left(X_{n}\right),\left(\lambda_{n}\right)$ and $\left(\beta_{n}\right)$ where $\left(X_{n}\right)$ is quasi- $\beta$-power increasing sequence as taken in the statement of the theorem the following condition holds

$$
n \beta_{n} X_{n}=\mathrm{O}(1) \text { as } n \rightarrow \infty \text {. }
$$

and

$$
\sum_{n=1}^{\infty} \beta_{n} X_{n}<\infty
$$

Lemma 4.2: (BOR [2]) If condition (2.6) and (2.7) are satisfied then we have

$$
\Delta\left(\frac{P_{n}}{n^{2} p_{n}}\right)=\mathrm{O}\left(\frac{1}{n^{2}}\right)
$$

Lemma 4.3: (BOR [2]) If the condition (2.1)-(2.4) are satisfied, then we have

$$
\begin{aligned}
\lambda_{n} & =\mathrm{O}(1) \\
\Delta \lambda_{n} & =\mathrm{O}\left(\frac{1}{n}\right)
\end{aligned}
$$

\subsection{PROOF OF THE THEOREM:}

Let $\left(T_{n}\right)$ be the sequence of $\left(\bar{N}, p_{n}\right)$ means of the series $\sum_{n=1}^{\infty} \frac{a_{n} P_{n} \lambda_{n}}{n p_{n}}$. Then we have

$$
\begin{aligned}
T_{n}=\frac{1}{P_{n}} \sum_{v=1}^{n} p_{v} \sum_{n=1}^{v} \frac{a_{r} P_{r} \lambda_{r}}{r p_{r}} \\
=\frac{1}{P_{n}} \sum_{v=1}^{n}\left(P_{v}-P_{v-1}\right) \frac{a_{v} P_{v} \lambda_{v}}{v p_{v}}
\end{aligned}
$$

then, for $n \geq 1$ 


$$
\begin{gathered}
T_{n}-T_{n-1}=\frac{p_{n}}{P_{n} P_{n-1}} \sum_{v=1}^{n} \frac{P_{v-1} P_{v} a_{v} v \lambda_{v}}{v p_{v}} \\
=\frac{p_{n}}{P_{n} P_{n-1}} \sum_{v=1}^{n} \frac{P_{v-1} P_{v} a_{v} v \lambda_{v}}{v^{2} p_{v}}
\end{gathered}
$$

Using Abel's transformation, we get

$$
\begin{gathered}
T_{n}-T_{n-1}=\frac{p_{n}}{P_{n} P_{n-1}} \sum_{v=1}^{n} \Delta\left(\frac{P_{v-1} P_{v} \lambda_{v}}{v^{2} p_{v}}\right) \sum_{r=1}^{n} r a_{r}+\frac{\lambda_{n}}{n^{2}} \sum_{v=1}^{n} v a_{v} \\
=\frac{p_{n}}{P_{n} P_{n-1}} \sum_{v=1}^{n-1} \frac{P_{v}}{p_{v}}(v+1) t_{v} p_{v} \frac{\lambda_{v}}{v^{2}}+\frac{p_{n}}{P_{n} P_{n-1}} \sum_{v=1}^{n-1} P_{v} p_{v} \Delta \lambda_{v}(v+1) \frac{t_{v}}{v^{2} p_{v}} \\
\quad-\frac{p_{n}}{P_{n} P_{n-1}} \sum_{v=1}^{n-1} p_{v} \lambda_{v+1}(v+1) t_{v}\left(\frac{P_{v}}{v^{2} p_{v}}\right)+\frac{\lambda_{n} t_{n}(n+1)}{n^{2}} \\
=T_{n, 1}+T_{n, 2}+T_{n, 3}+T_{n, 4} \quad \text { (say) }
\end{gathered}
$$

To complete the proof of the theorem by Minkowski's inequality, it is sufficient to show that

$$
\sum_{n=1}^{\infty}\left(\frac{P_{n}}{p_{n}}\right)^{\delta k+k-1}\left|T_{n, r}\right|^{k}<\infty, \text { for } r=1,2,3,4
$$

Now, applying Hölder's inequality, we have that

$$
\begin{aligned}
& \sum_{n=2}^{m+1}\left(\frac{P_{n}}{p_{n}}\right)^{\delta k+k-1}\left|T_{n, 1}\right|^{k} \\
& =\mathrm{O}(1) \sum_{n=2}^{m+1}\left(\frac{P_{n}}{p_{n}}\right)^{\delta k-1} \frac{1}{P_{n-1}^{k}}\left\{\sum_{v=1}^{n-1} \frac{P_{v}}{p_{v}} p_{v}\left|t_{v} \| \lambda_{v}\right| \frac{1}{v}\right\}^{k} \\
& =\mathrm{O}(1) \sum_{n=2}^{m+1}\left(\frac{P_{n}}{p_{n}}\right)^{\delta k-1} \frac{1}{P_{n-1}} \sum_{v=1}^{n=1}\left(\frac{P_{v}}{p_{v}}\right)^{k} p_{v}\left|t_{v}\right|^{k}\left|\lambda_{v}\right|^{k} \frac{1}{v^{k}}\left[\frac{1}{P_{n-1}} \sum_{v=1}^{n-1} p_{v}\right]^{k-1} \\
& =\mathrm{O}(1) \sum_{v=1}^{m}\left(\frac{P_{v}}{p_{v}}\right)^{k} p_{v}\left|t_{v}\right|^{k}\left|\lambda_{v}\right|^{k} \frac{1}{v^{k}} \sum_{n=v+1}^{m+1}\left(\frac{P_{n}}{p_{n}}\right)^{\delta k-1} \frac{1}{P_{n-1}} \\
& =\mathrm{O}(1) \sum_{v=1}^{m}\left(\frac{P_{v}}{p_{v}}\right)\left|\lambda_{v}\right|^{k-1}\left|\lambda_{v}\right| p_{v}\left|t_{v}\right|^{k} \frac{1}{v^{k}} \frac{1}{P_{v}}\left(\frac{P_{v}}{p_{v}}\right)^{\delta k} \\
& =\mathrm{O}(1) \sum_{v=1}^{m}\left(\frac{P_{v}}{p_{v}}\right)^{k-1}\left|\lambda_{v} \| t_{v}\right|^{k} \frac{1}{v^{k}}\left(\frac{P_{v}}{p_{v}}\right)^{\delta k} \\
& =\mathrm{O}(1) \sum_{v=1}^{m}\left(\frac{P_{v}}{p_{v}}\right)^{\delta k} v^{k-1} \frac{1}{v^{k}}\left|\lambda_{v} \| t_{v}\right|^{k} \\
& =\mathrm{O}(1) \sum_{v=1}^{m}\left|\lambda_{v}\right|\left(\frac{P_{v}}{p_{v}}\right)^{\delta k} \frac{\left|t_{v}\right|^{k}}{v}
\end{aligned}
$$




$$
\begin{aligned}
& =\mathrm{O}(1) \sum_{v=1}^{m-1} \Delta\left|\lambda_{v}\right| \sum_{r=1}^{v}\left(\frac{P_{r}}{p_{r}}\right)^{\delta k} \frac{\left|t_{r}\right|^{k}}{r}+\mathrm{O}(1)\left|\lambda_{m}\right| \sum_{v=1}^{m}\left(\frac{P_{v}}{p_{v}}\right) \frac{\left|t_{v}\right|^{k}}{v} \\
& =\mathrm{O}(1) \sum_{v=1}^{m-1}\left|\Delta \lambda_{v}\right| X_{v}|+\mathrm{O}(1)| \lambda_{m} \mid X_{m} \\
& =\mathrm{O}(1) \sum_{v=1}^{m-1} \beta_{v} X_{v}+\mathrm{O}(1)\left|\lambda_{m}\right| X_{m} \\
& =\mathrm{O}(1) \text { as } m \rightarrow \infty \\
& \text { Next } \\
& \sum_{n=2}^{m+1}\left(\frac{P_{n}}{p_{n}}\right)^{\delta k+k-1}\left|T_{n, 2}\right|^{k} \\
& =\mathrm{O}(1) \sum_{n=2}^{m+1}\left(\frac{P_{n}}{p_{n}}\right)^{\delta k-1} \frac{1}{P_{n-1}^{k}}\left\{\sum_{v=1}^{n-1} \frac{P_{v}}{p_{v}}\left|\Delta \lambda_{v} \| t_{v}\right|\right\}^{k} \\
& =\mathrm{O}(1) \sum_{n=2}^{m+1}\left(\frac{P_{n}}{p_{n}}\right)^{\delta k-1} \frac{1}{P_{n-1}} \sum_{v=1}^{n-1}\left(\frac{P_{v}}{p_{v}}\right)^{k}\left|\Delta \lambda_{v}\right|^{k}\left|t_{v}\right|^{k} p_{v}\left\{\frac{1}{P_{n-1}} \sum_{v=1}^{n-1} p_{v}\right\}^{k-1} \\
& =\mathrm{O}(1) \sum_{v=1}^{m}\left(\frac{P_{v}}{p_{v}}\right)^{k} p_{v}\left|t_{v}\right|^{k}\left|\Delta \lambda_{v}\right|^{k} \sum_{n=v+1}^{m+1}\left(\frac{P_{n}}{p_{n}}\right)^{\delta k-1} \frac{1}{P_{n-1}} \\
& =\mathrm{O}(1) \sum_{v=1}^{m}\left(\frac{P_{v}}{p_{v}}\right)^{k-1}\left|\Delta \lambda_{v}\right|^{k} p_{v}\left|t_{v}\right|^{k}\left(\frac{P_{v}}{p_{v}}\right)^{\delta k} \\
& =\mathrm{O}(1) \sum_{v=1}^{m}\left(\frac{P_{v}}{p_{v}}\right)^{k-1}\left|\Delta \lambda_{v}\right|^{k}\left|t_{v}\right|^{k}\left(\frac{P_{v}}{p_{v}}\right)^{\delta k} \\
& =\mathrm{O}(1) \sum_{v=1}^{m}\left(\frac{P_{v}}{p_{v}}\right)^{\delta k}\left(\frac{P_{v}}{p_{v}}\right)^{k-1}\left|\Delta \lambda_{v}\right|^{k-1}\left|\Delta \lambda_{v} \| t_{v}\right|^{k} \\
& =\mathrm{O}(1) \sum_{v=1}^{m}\left(\frac{P_{v}}{p_{v}}\right)^{\delta k} v^{k-1} \frac{1}{v^{k-1}}\left|\Delta \lambda_{v} \| t_{v}\right|^{k} \\
& =\mathrm{O}(1) \sum_{v=1}^{m} \beta_{v}\left(\frac{P_{v}}{p_{v}}\right)^{\delta k}\left|t_{v}\right|^{k} \\
& =\mathrm{O}(1) \sum_{v=1}^{m} v \beta_{v}\left(\frac{P_{v}}{p_{v}}\right)^{\delta k} \frac{\left|t_{v}\right|^{k}}{v} \\
& =\mathrm{O}(1) \sum_{v=1}^{m-1} \Delta\left(v \beta_{v}\right) \sum_{r=1}^{v}\left(\frac{P_{v}}{p_{r}}\right)^{\delta k} \frac{\left|t_{r}\right|^{k}}{r}+\mathrm{O}(1) m \beta_{m} \sum_{v=1}^{n}\left(\frac{P_{v}}{p_{v}}\right)^{\delta k} \frac{\left|t_{v}\right|^{k}}{v} \\
& =\mathrm{O}(1) \sum_{v=1}^{m-1} v\left|\Delta \beta_{v}\right| X_{v}+\mathrm{O}(1) \sum_{v=12}^{m-1} \beta_{v} X_{v}+\mathrm{O}(1) m \beta_{m} X_{m}
\end{aligned}
$$


$=\mathrm{O}(1)$ as $m \rightarrow \infty$

Next,

$\sum_{n=2}^{m+1}\left(\frac{P_{n}}{p_{n}}\right)^{\delta k+k-1}\left|T_{n, 3}\right|^{k}$

$=\mathrm{O}(1) \sum_{n=2}^{m+1}\left(\frac{P_{n}}{p_{n}}\right)^{\delta k-1} \frac{1}{P_{n-1}^{k}}\left\{\sum_{v=1}^{n-1} P_{v}\left|\lambda_{v+1} \| t_{v}\right| \frac{1}{v}\left(\frac{v+1}{v}\right)\right\}^{k}$

$=\mathrm{O}(1) \sum_{n=2}^{m+1}\left(\frac{P_{n}}{p_{n}}\right)^{\delta k-1} \frac{1}{P_{n-1}^{k}}\left\{\sum_{v=1}^{n-1} \frac{P_{v}}{p_{v}} p_{v}\left|\lambda_{v+1}\right| \frac{1}{v}\left|t_{v}\right|\right\}^{k}$

$=\mathrm{O}(1) \sum_{n=2}^{m+1}\left(\frac{P_{n}}{p_{n}}\right)^{\delta k-1} \frac{1}{P_{n-1}} \sum_{v=1}^{n-1}\left(\frac{P_{v}}{p_{v}}\right)^{k} p_{v} \frac{1}{v^{k}}\left|\lambda_{v+1}\right|^{k}\left|t_{v}\right|^{k}\left\{\frac{1}{P_{n-1}} \sum_{v=1}^{n-1} p_{v}\right\}^{k-1}$

$=\mathrm{O}(1) \sum_{v=1}^{m}\left(\frac{P_{v}}{p_{v}}\right)^{k} p_{v} \frac{1}{v^{k}}\left|\lambda_{v+1}\right|^{k-1}\left|\lambda_{v+1}\right|\left|\mathrm{t}_{v}\right|^{k}\left\{\sum_{n=v+1}^{m+1}\left(\frac{P_{n}}{p_{n}}\right)^{\delta k-1} \frac{1}{P_{n-1}}\right\}$

$=\mathrm{O}(1) \sum_{v=1}^{m}\left(\frac{P_{v}}{p_{v}}\right)^{k-1} \frac{1}{v^{k}}\left|\lambda_{v+1} \| t_{v}\right|^{k}\left(\frac{P_{v}}{p_{v}}\right)^{\delta k}$

$=\mathrm{O}(1) \sum_{v=1}^{m}\left(\frac{P_{v}}{p_{v}}\right)^{\delta k} v^{k-1} \frac{1}{v^{k}}\left|\lambda_{v+1} \| t_{v}\right|^{k}$

$=\mathrm{O}(1) \sum_{v=1}^{m}\left(\frac{P_{v}}{p_{v}}\right)^{\delta k}\left|\lambda_{v+1}\right| \frac{\left|t_{v}\right|^{k}}{v}$

$=\mathrm{O}(1) \sum_{v=1}^{m-1} \Delta\left|\lambda_{v+1}\right| \sum_{r=1}^{v}\left(\frac{P_{r}}{p_{r}}\right)^{\delta k} \frac{\left|t_{r}\right|^{k}}{r}+\mathrm{O}(1)\left|\lambda_{m+1}\right| \sum_{v=1}^{m}\left(\frac{P_{v}}{p_{v}}\right)^{\delta k} \frac{\left|t_{v}\right|^{k}}{v}$

$=\mathrm{O}(1) \sum_{v=1}^{m-1}\left|\Delta \lambda_{v+1}\right| X_{v}+\mathrm{O}(1)\left|\lambda_{m+1}\right| X_{m}$

$=\mathrm{O}(1) \sum_{v=1}^{m-1}\left|\Delta \lambda_{v+1}\right| X_{v}+\mathrm{O}(1)\left|\lambda_{m+1}\right| X_{m}$

$=\mathrm{O}(1) \sum_{v=1}^{m} \beta_{v} X_{v}+\mathrm{O}(1)\left|\lambda_{m+1}\right| X_{m+1}$

$=\mathrm{O}(1)$ as $m \rightarrow \infty$

Finally

$\sum_{n=1}^{m}\left(\frac{P_{n}}{p_{n}}\right)^{\delta k+k-1}\left|T_{n, 4}\right|^{k}$

$=\mathrm{O}(1) \sum_{n=1}^{m}\left(\frac{P_{n}}{p_{n}}\right)^{\delta k}\left(\frac{P_{n}}{p_{n}}\right)^{k-1}\left(\frac{n+1}{n}\right)^{k} \frac{1}{n^{k}}\left|\lambda_{n}\right|^{k}\left|t_{n}\right|^{k}$ 


$$
\begin{aligned}
& =\mathrm{O}(1) \sum_{n=1}^{m}\left(\frac{P_{n}}{p_{n}}\right)^{\delta k} n^{k-1} \frac{1}{n^{k}}\left|\lambda_{n}\right|^{k-1}\left|t_{n}\right|^{k}\left|\lambda_{n}\right| \\
& =\mathrm{O}(1) \sum_{n=1}^{m}\left|\lambda_{m}\right|\left(\frac{P_{n}}{p_{n}}\right)^{\delta k} \frac{\left|t_{n}\right|^{k}}{n} \\
& =\mathrm{O}(1) \text { as } m \rightarrow \infty .
\end{aligned}
$$

Therefore, we get that

$$
\sum_{n=1}^{m}\left(\frac{P_{n}}{p_{n}}\right)^{\delta k+k-1}\left|T_{n, r}\right|^{k}=\mathrm{O}(1) \text { as } m \rightarrow \infty \text { for } r=1,2,3,4
$$

This completes the proof of the theorem.

\section{Corollary:}

Our theorem has following results as a corollary.

Corollary 6.1: Taking $\delta=0$ in theorem 4.1, we get Theorem 2.1 as a corollary. Since for $\delta=0,\left|\bar{N}, p_{n}, \delta\right|_{k}$ summability reduces to $\left|\bar{N}, p_{n}\right|_{k}$-summability.

\section{Conclusion:}

Our theorem have the more general result rather than any previous known results. So our theorem enrich the literature of summability theory.

\section{Acknowledgements:}

I am very thankful to Dr. B.K. Singh, (Professor and Head of the Dept. of Mathematics, IFTM Uni. Modradabad, U.P., India) whose great inspirations lead me to complete this paper.

\section{References:}

[1] BOR, H.; On two summability methods, Math. Proc. Camb. Philos Soc. 97 (1985).

[2] BOR, H.; Absolute summability factors for infinite series. Indian J. Pure and Appl. Math. 19 (1988)

[3] BOR, H.; On local property of $\left|\bar{N}, p_{n}, \delta\right|_{k}$-summability of factored Fourier series, J. Math. Anal. Appl., 179 (1993).

[4] BOR, H.; A study on absolute Riesz summability factors, Rend. Circ. Mat. Palcrmo, 56 (2007).

[5] BOR, H. On an application of almost increasing sequence. Math. Commun. Vol., 15 (2010).

[6] Flett, T.M.; On an extension of absolute summability and some theorems of Littlewood and Paley, Proc. London Math. Soc. 7 (1957).

[7] Flett, T.M.; Some more theorems concerning the absolute summability of Fourier series and power series, Proc. London Math Soc. 8 (1958).

[8] Hardy, G.H.; Divergent Series, Oxford University Press, Oxford, 1947.

[9] Leindler, L.; A new application of quasi power increasing sequence, Bull. Math. Debrecen, 58 (2001). 